\title{
Dynamic Channel Allocation Control with thresholds in Wireless Cellular Networks using Simpy
}

\author{
Yang Cao*, Cheul Woo Ro** \\ *Dep. of Information \& Technology. Eastern Liaoning University, Dandong, CHINA \\ **Dep. of Computer. Eng. Silla University, Busan, KOREA
}

\begin{abstract}
New and handoff calls control mechanisms are the key point to wireless cellular networks. In this paper, we present an adaptive algorithm for dynamic channel allocation scheme with guard channels and also with handoff calls waiting queue ensuring that handoff calls take priority over new calls. Our goal is to find better tradeoff between handoffs and new calls blocking probabilities in order to achieve more efficient channel utilization. Simpy is a Python based discrete event simulation system. We use Simpy to build our simulation models to get analytical data.
\end{abstract}

Keywords: Cellular Network, Dynamic Guard Channels, Handoff Calls, Simpy

\section{INTRODUCTION}

In Wireless Cellular Networks it is still an accelerated research to handle handoff calls in an appropriate way. The mobility of subscribers especially at the boundary of cells causes great annoyances [1]. Handoff call dropping, due to lack of wireless resources, gets more concerns than the rejection of a new call—call blocking [2].

A better way to solve this kind of problems is the use of guard channel policy which provides a priority for handoff calls over new calls [3]. Under this policy, a handoff call is admitted as long as there is a free nominal or guard channel, while a new call request is admitted only if there is a free nominal channel. Handoff calls are privileged over new call requests. It is the key point to decide the appropriate number of guard channels in order to minimize the call dropping rate and at the same time to avoid waste of channels by blocking new calls [4]. Due to the variability of traffic load, scheme of dynamically adapting guard channels gains more attention.

In this paper, we present an adaptive algorithm which can dynamically adjust the number of guard channels with some thresholds restraining the handoff calls dropping probability (Pdh), at the same time using handoff calls waiting queue for further ensuring that handoff calls take priority over new calls. We presume that each cell of the network has the same architecture and of same capacity, so we just need to focus on one cell. We compare our methods with fixed guard channel policy as well as no guard channel policy. The numerical results prove that our algorithm can achieve optimal performance--better tradeoff between handoff calls dropping

\footnotetext{
* Corresponding author, Email: cwro@silla.ac.kr Manuscript received Mar. 30, 2012; revised Jun 15, 2012; accepted Jun 18, 2012
}

probability (Pdh) and new calls blocking probability (Pbn), meanwhile, guarantee higher resource utilization. Simpy is a Python-based discrete event simulation system that models active components such as messages, customers, trucks, planes by parallel processes [5]. We use the popular simulation tool-Simpy to build our simulation models to get analytical data.

The rest of the paper is organized as follows. In Section 2, we discuss the previous work in the literature on channel reservation policy. The system modeling is discussed in Section 3. We give numerical analysis on performance in Section 4, and conclude in Section 5.

\section{RELATED WORK}

\subsection{Handoff Strategies}

To improve the quality of service (QoS), various handoff strategies are studied to prioritize handoff calls over new calls, which can mainly fall into two categories: guard channel and handoff queue [6].

The study of guard channels (GC) was first started by Hong and Rappaport [6]. Then an extension of GC considering multiple service types are presented by Li et al [7]. The concept of Multiple Fractional Guard Channel (MFGC) was proposed by Heredia [8]. Those schemes decreased dropping probability of handoff calls to a great extent.

Handoff queue scheme is another commonly used handoffs prioritization strategy which puts the handoff calls in the waiting queue when all channels in a BS are occupied. When a channel is released, the handoff calls in the queue gains the channel prior to a new call [9].

The schemes mentioned above greatly reduce the $\boldsymbol{P} \boldsymbol{b} \boldsymbol{h}$ while sacrificing the Pbn. Recent years, various dynamic guard 
channel schemes are discussed [4],[10]-[13]. Normally they use thresholds to periodically monitor the $\boldsymbol{P} \boldsymbol{b} \boldsymbol{h}$, and dynamically adjust the number of guard channels. The aim is to keep the $\boldsymbol{P b h}$ under reasonable limitation meanwhile decreasing as possible the $\boldsymbol{P b n}$ in order to get higher system performance. In this paper, we also monitor the changes of $\boldsymbol{P} \boldsymbol{d h}$ timely to keep it within some limitations (thresholds). We present an adaptive algorithm for guard channel reservation and at the same time adopt a FIFO queue for handoff calls in each BS in order to get better both $\boldsymbol{P b h}$ and $\boldsymbol{P b n}$.

\subsection{General Distribution}

In order to construct a more realistic model of Internet traffic sources, we employ self-similar traffic models. Detailed discussion of self-similarity in time series data and the accompanying statistical tests are found in [14]. Normally the time series distributions of self-similar traffic are heavy-tailed, while Pareto distribution is the simplest heavy-tailed distribution. The Pareto distribution is power-law over its entire range, its probability density function is given by [15]:

$$
p(x)=\alpha k^{\alpha} x^{-\alpha-1}, \quad \alpha, k>0, \quad x \geq k .
$$

Its distribution function has the form

$$
F(x)=P[X \leq x]=1-(k / x)^{\alpha}
$$

Here $\mathrm{k}$ means the smallest possible value of the random variable (location parameter). With $\alpha$ (shape parameter) decreases, a large portion of the probability mass is present in the tail of the distribution. In practical terms a random variable that follows a heavy-tailed distribution can be extremely large values with non-negligible probability. We use Exponential and Pareto distribution to simulate the call arrival rate and the calls service time, respectively.

\subsection{Discrete Event Simulation and Simpy}

Discrete Event Simulation (DES) was first introduced in the 1950s to solve business problems. It is one way of modeling, simulating, and analyzing a system to observe the behavior of that system. During the simulation process the models are executed over times in order to generate results which are then the basis for future decision making [16][17]. Mechanisms proposed for carrying out DES involve event-based, activity-based, proves-based and three-phase approaches [18].

Simpy is one of discrete events simulation systems based on Python. The simulation is process-oriented, which means that it provides the modeler a number of tools including processes to model active entities, resource facilities (Resources, Levels, and Stores) and ways of recording results [19]. Simpy allows us conveniently and easily to create models of discrete event systems [20].

\section{SYSTEM MODELING}

As we all know, wireless channel allocation policies may have significant impact on the performance of the system, for example, the new call and handoff calls blocking probabilities, the system utilization, etc. A well-designed policy should take full account of these conditions. We present several kinds of models representing wireless cellular networks with different allocation policies. We then compare our adaptive algorithm with these policies. All the models we've built adopt a handoff call waiting queue for more emphasis on handoffs.

\subsection{Policy 1. No Reserved Guard Channels}

The kind of model runs like that when a new call (NC) is attempting in a cell covered by a BS, the NC is connected if an idle channel is available in the cell. Otherwise, the new call is blocked. When a handoff call (HC) is moving from one cell to another, it is connected if an idle channel is available in the cell. Otherwise, if the calls waiting queue is not full, it will arranged to the queue waiting for idle channels. If the queue is full, the HC will be dropped.

\subsection{Policy 2. Fixed Number of Guard Channels}

The kind of models runs like that the processing of new call is similar to the former model. A fixed number of channels exclusively for HCs are reserved as guard channels to reduce the dropping probability of HCs since it is a viral measure of wireless network. Policy 2.1 Policy 2.3 keep 1 3 fixed guard channels respectively. A call waiting queue also kept for handoff calls. According to our former work [21], we get better GoS when taking 2 guard channels. So in these models we choose 1 3 guard channels.

\subsection{Policy 3. Adaptive Guard Channels with thresholds}

This kind of model runs like the former ones except that the system adjusts the number of guard channels $(\boldsymbol{g})$ automatically according to the current Pdh. In this paper, we give the dynamic channel control with thresholds as follows: If the $\boldsymbol{P d h}$ is below the given minimum threshold Tmin, which means we needn't keep so many guard channels for handoffs, then the number of guard channels decrease until the current $\boldsymbol{P d h}$ is exceeding the given maximum threshold Tmax. Then we need to increase the number of guard channels to make sure it is below the Tmax. There are also limitation threshold values for $\boldsymbol{g}$. The value of the $\boldsymbol{g}$ is between $\left[\boldsymbol{g} \_\boldsymbol{m i n}, \boldsymbol{g} \_\boldsymbol{m a x}\right]$. The benefit of this adaptation is that we keep the $\boldsymbol{P d} \boldsymbol{h}$ within some thresholds restriction while the $\boldsymbol{P} \boldsymbol{b n}$ is reduced which can improve the system performance. The algorithm is shown below:

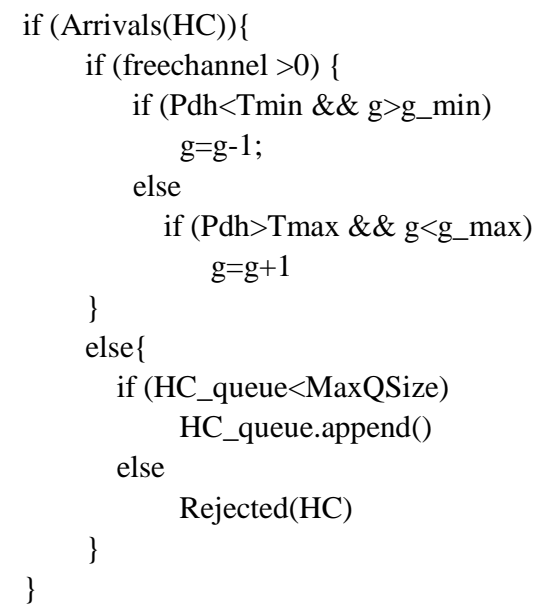




\section{MODEL ANALYSIS}

\subsection{Measures of Interest}

In order to obtain the interested measures numerically from the models, Simpy simulation package is adopted. We consider each cell with a total of 50 free channels and the size of handoffs queue is 3 . We assume that all calls arriving process is assumed to be exponentially distributed with mean Hrate and Nrate for handoffs and new calls respectively, while the call duration is Pareto distributed with the shape parameter $a$. In the simulation we keep that Nrate/Hrate $=5 / 1$ and $a=1.5$.

- $\quad$ NC blocking probability (Pbn)

We get $\boldsymbol{P} \boldsymbol{b n}$ value by calculating the probability:

NRej['new call']/ NArrv['new call']

- $\quad$ HC dropping probability (Pdh)

We get $\boldsymbol{P d h}$ value by calculating the probability: NRej['handoff']/ NArrv['handoff']

- $\quad$ Grade of Service(GoS)

We then define a formula to represent grade of service for system performance evaluation, $W$ is the weight to take use of Pbn. Here we let $W=0.1$ in order to focus heavily on $\mathrm{HC}$ dropping rate. The formula is as the following:

$$
G o S=P d h+W * P b n
$$

\subsection{Input Data}

We get the numerical results by giving some different input parameters. Table 1 lists the input data we give to run the simulation. The simulation time is set to be 10000.0 for better accuracy.

Table 1. Input data

\begin{tabular}{|c|c|c|}
\hline Parameter & Meaning & Value \\
\hline C & $\begin{array}{l}\text { Number of total } \\
\text { channels }\end{array}$ & 50 \\
\hline$g$ & $\begin{array}{l}\text { Number of guard } \\
\text { channels }\end{array}$ & $\begin{array}{l}\text { Policy 1: } 0 \\
\text { Policy 2.1: } 1 \\
\text { Policy 2.2: } 2 \\
\text { Policy 2.3: } 3 \\
\text { Policy 3: adaptive }\end{array}$ \\
\hline $\begin{array}{l}\text { Nrate } \\
\text { Hrate }\end{array}$ & $\begin{array}{l}\text { New call arrival rate } \\
\text { Handoff call arrival rate }\end{array}$ & Nrate $/$ Hrate $=5 / 1$ \\
\hline$a$ & $\begin{array}{l}\text { Para. of function } \\
\text { paretovariate() }\end{array}$ & 1.5 \\
\hline Tmax & Upper limit of $P d h$ & 0.009 \\
\hline Tmin & Lower limit of $P d h$ & 0.006 \\
\hline g_max & Upper limit of $g$ & 10 \\
\hline g_min & Lower limit of $g$ & 0 \\
\hline$W$ & $\begin{array}{l}\text { Weighting factor of } \\
\text { GoS }\end{array}$ & 0.1 \\
\hline
\end{tabular}

\subsection{Numerical Results}

Figure 1 shows different $\boldsymbol{P d h}$ of those models with different new calls arriving rate Nrate. We can see that with the number of arriving calls increases, Policy 3 shows best results of $\boldsymbol{P d h}$ than other policies, and with the adaptive function of guard channels it can keep Pdh under the threshold limitation.

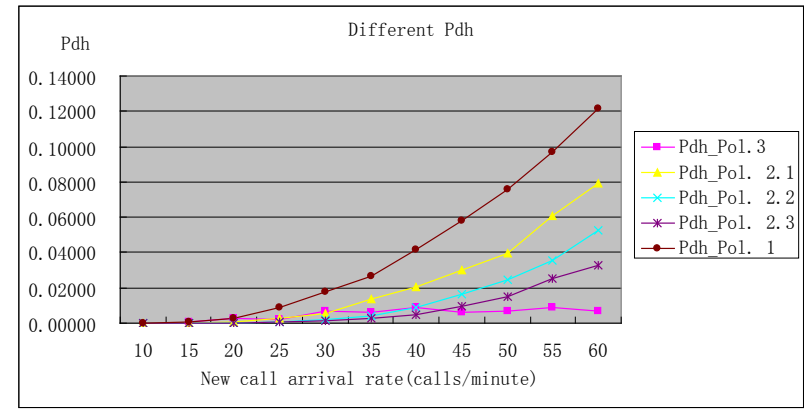

Fig. 1. Handoff call dropping probability.

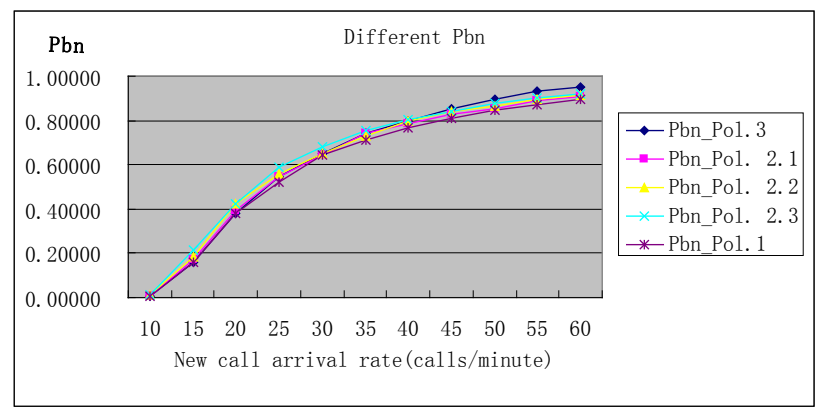

Fig. 2. New call Blocking probability.

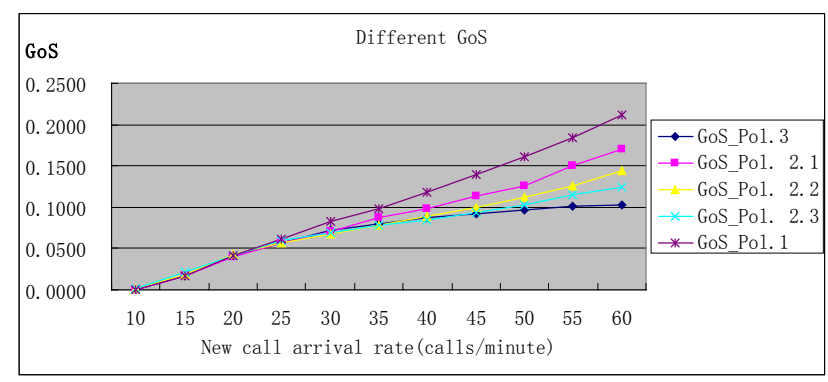

Fig. 3. Grade of Service.

Figure 2 shows new call blocking probabilities of those models with different new calls arriving rate Nrate. There are very vital differences between those policies. And because Policy 3 gives more priority to handoff calls, certainly it gets a little bit higher values of Pbn. Figure 3 shows different $\boldsymbol{G o S}$ of those models we calculated with corresponding $\boldsymbol{P d h}$ and $\boldsymbol{P b n}$ values. We can see Policy 3 gets the best system performance. In those figures, Pol.1 means Policy 1, Pol. 2.1 means Policy 2.1 , and so on.

As we can see, with the increasing of Nrate, handoff calls dropping probability arise correspondingly. Among which Policy 2.3 with more guard channels reserved keeps lowest $\boldsymbol{P d h}$, while with the expense of highest $\boldsymbol{P} \boldsymbol{b n}$. For our adaptive algorithm, the $\boldsymbol{P d h}$ are with lower value and can be kept under the given threshold, while the $\boldsymbol{P b n}$ still kept in lower values. So we can get better system performance, like $\boldsymbol{G o S}$.

\section{CONCLUSION}

In this paper, several channel allocation models with different policies have been constructed to get performance 
analysis using Simpy simulation package. We present an adaptive algorithm which aims at keeping balance between $\boldsymbol{P d h}$ and $\boldsymbol{P b n}$ for better system performance. We compare our new model to those models with different allocation policies and analyze their performances such as $\boldsymbol{P d h}, \boldsymbol{P b n}$, and $\boldsymbol{G o S}$ by giving some input parameters. According to the final results we can declare that system using adaptive guard channel policy is more efficient than those other scheduling policies. Our future work will extend this algorithm to add and analyze the utilization of radio resources

\section{REFERENCES}

[1] T.V. Do, "A new computational algorithm for retrial queues to cellular mobile systems with guard channels", Computers \& Industrial Engineering (59) 865-872, 2010.

[2] S. Limm, G. Cao, C. R. Das, "A differential bandwidth reservation policy for multimedia wireless networks", Parallel Processing Workshops, International Conference, pp. 447-452, 2001.

[3] R.Guerin, "Queueing-blocking system with two arrival streams and guard channels", IEEE Transactions on Communications, 36(2), 153-163, 1988.

[4] Y. Zhang and D. Liu, "An adaptive algorithm for call admission control in wireless networks", Proceedings of the IEEE Global Communications Conference, pp. 3628-3632, 2001.

[5] http://simpy.sourceforge.net

[6] T. Rappaport, "Wireless Communications Principles and Practice Second Edition", Prentice Hall, pp.34, 2002.

[7] B. Li, C. Lin, and S. T. Chanson, "Analysis of a hybrid cutoff priority scheme for multiple classes of traffic in multimedia wireless networks", ACM/Baltzer Wireless Networks Journal, vol. 4, no. 4, pp. 279-290, 1998.

[8] H. Heredia-Ureta, F.A. Cruz-Pérez and L. Ortigoza-Guerrero, "Multiple fractional channel reservation for optimum system capcity in multi-service cellular networks", Electronic Letters, vol. 39, pp. 133-134, 2003.

[9] M. Oliver, J. Borras, "Performance evaluation of variable reservation policies for hand-off prioritization in mobile networks", Infocom'99 Proceedings of IEEE 3, pp. 1187-1194, 1999.

[10] Xue-Ping Wang, Jian-Li Zheng, Wei Zeng, Gen-Du Zhang, "A probability-based adaptive algorithm for call admission control in wireless network", International Conference on Computer Networks and Mobile Computing, pp. 197-204, 2003.

[11] Wei YY, Lin C, F.Y. Ren, "Dynamic handoff scheme in differentiated QoS wireless multimedia networks", Computer Communications, vol.27, pp. 1001-1011, 2004.
[12] M. Salamah, H. Lababidi, "Dynamically adaptive channel reservation scheme for cellular networks", Computer Networks, pp. 787-796, 2005.

[13] A. Saddoud, L. Fourati Chaari, L. Kamoun, "Adaptive algorithm for Admission Control in wireless networks", Communications and Networking (ComNet), 2010 Second International Conference, pp. 1-6, 2010.

[14] Walter Willinger, Murad S. Taqqu, Will E. Leland, and Daniel V. Wilson, "Self-similarity in high-speed packet traffic: Analysis and modeling of Ethernet traffic measurements", Statistical Science, 10(1):67--85, 1995.

[15] M. E. Crovella, A. Bestavros, "Self-Similarity in World Wide Web Traffic: Evidence and Possible Causes", IEEE/ACM Transactions on Networking, vol. 5, no. 6, pp. 835-846, 1997.

[16] P. Ball, "Introduction to Discrete Event Simulation", 2nd DYCOMANS workshop on "Management and Control: Tools in Action" in the Algarve, Portugal, pp. 367-376, 1996.

[17] E. Babulak, M. Wang, "Discrete Event Simulation: State of the Art", Discrete Event Simulations, Sciyo, pp. 1-9, 2010.

[18] M. Pidd, "Computer simulation in management science fourth edition", Wiley, 1998.

[19] T. Vignaux, K. Muller, B. Helmbold, "The SimPy Manual", http://simpy.sourceforge.net/SimPyDocs/Manual.html, 2007.

[20] N.S. Matloff, "Introduction to Discrete-Event Simulation and the SimPy Language", 2008

[21] Yang Cao, Cheul Woo Ro, "Non-Markovian SPN Model for Wireless Networks", Proceeding of ICCC, 2010.

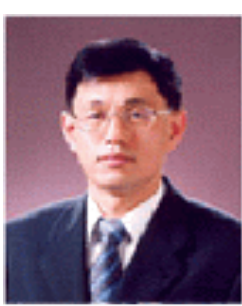

Cheul Woo Ro

Refer to IJOC, Vol.4, No. 3, 2008.9

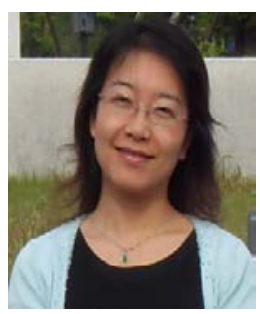

Yang Cao

She received the M.S in Belgium in 2003. She works at Eastern Liaoning University in China. She is currently a $\mathrm{PhD}$ candidate in the department of computer engineering at Silla University. Her main research interests include modeling and analysis of communication

systems. 